\title{
A Novel Molecular Indicator for Inhibitor Development in Haemophilia A
}

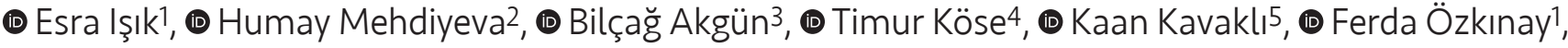 \\ (D) Tahir Atik1
}

'Ege University Faculty of Medicine, Department of Pediatrics, Division of Genetics, İzmir, Turkey

2Ege University Faculty of Medicine, Department of Pediatrics, İzmir, Turkey

3izmir University of Economics, Faculty of Medicine, Department of Medical Genetics, İzmir, Turkey

${ }^{4}$ Ege University Faculty of Medicine, Department of Biostatistics and Medical Informatics, İzmir, Turkey

${ }^{5}$ Ege University Faculty of Medicine, Department of Pediatrics, Division of Hematology, İzmir, Turkey

\begin{abstract}
Aim: Previous studies have reported inhibitor development (ID) risk in those patients who have hemophilia A (HA) with missense mutations to be $3-10 \%$. We investigated the association between ID risk and various features of missense mutations; including the impact directly related to amino acid group change.

Materials and Methods: Missense mutations in the F8 gene, clinical findings of the patients including severity of HA, and ID status were obtained from the F8 gene variant database (http://www.factorviii-db.org/). Twenty amino acids were then classified into groups according to their side chains. All information regarding each specific mutation, as well as any impact of the mutation on the amino acid group change, was recorded. Additionally, localization (at which domain) of any changed amino acid in the F8 protein was noted. Combined Annotation Dependent Depletion (CADD), Rare Exome Variant Ensemble Learner (REVEL), Mendelian Clinically Applicable Pathogenicity and Deleterious Annotation using Neural Networks scores were applied to identify a significant cut-off value indicative of ID.

Results: Three variations were identified that could be considered as useful in the prediction of ID in mild HA. The first being that among mild HA patients, $7.9 \%$ ( $n=70 / 883$ ) with mutations causing no amino acid group changes showed ID. This rate, however, was only $2.9 \%$ in patients with mutations leading to amino acid group changes. Secondly; in patients with mutations causing no amino acid group changes affecting A2, $A 3$ and C2 domains, ID risk was found to be higher than in patients with mutations leading to amino acid group changes. Thirdly; an association between ID and CADD and REVEL scores was observed.

Conclusion: In mild HA patients, the characteristics of missense mutations in terms of amino acid group changes, and CADD and REVEL scores could be of considerable utility in the prediction of ID risk.
\end{abstract}

Keywords: Hemophilia A, inhibitor, F8 gene, mutation, missense, interpretation

\section{Introduction}

The development of neutralizing antibodies against Factor VIII (FVIII) is a serious complication to the early stages of replacement therapy in hemophilia $A(H A)$. This is known as inhibitor development (ID). The overall incidence of ID is $20-30 \%$ (1). The mechanisms underlying ID are very complex and full understanding remains elusive. Risk factors that carry the potential for ID are classified simply 
into two groups: modifiable and unmodifiable. The most critical unmodifiable risk factors relate to the type of the causative mutation in the F8 gene, and the clinical severity (2-4). The major modifiable risk factors tend to be age at first treatment and the source of FVIII concentrate $(5,6)$.

To date, a number of studies have investigated the complex pathophysiological mechanisms leading to the development of FVIII inhibitors. Relationships between the type of F8 mutation and the risk of inhibitor formation have been extensively discussed in recent years. In 1995, Schwaab et al. (2) reported that cumulative incidences of ID in severe HA patients carrying large deletions, non-sense mutations, and intron 22 inversions were $35.7 \%, 38.4 \%$ and $34.4 \%$ respectively. However, in those patients with missense F8 mutations, this incidence was $4.3 \%$ (2). Several other studies on ID risk for missense F8 mutations confirmed these findings with reports of prevalence between 3 and $10 \%(7,8)$.

It is thought that the position and type of substitution of missense mutations may influence the risk of ID. The INSIGHT study analyzed the association between F8 mutation and ID in 1.112 patients with non-severe HA. From a total of 214 different F8 missense mutations identified, 19 were reported to be associated with ID (p.Leu412Phe, p.Arg531Cys, p.Arg593Cys, p.Asn618Ser, p.Pro1761Cln, p.Phe1775Val, p.Arg1781Gly, p.Pro1854Leu, p.Arg1997Trp, p.Asp2074Gly, p.Phe2101Cys, p.Tyr2105Cys, p.Arg2150His, p.Arg2159Cys, p.Glu2228Asp, p.Trp2229Cys, p.Val2232Ala, p.His2309Asp, p.Ter2333Cys) (9). It remains unclear why these specific missense mutations carry a higher risk for ID.

In this study, we investigated the association between ID risk and various features of missense mutations including those caused by amino acid group change.

\section{Materials and Methods}

Missense mutations in the F8 gene, along with the patients' clinical findings (including severity of $\mathrm{HA}$ and ID status) were obtained from the F8 gene variant database (http://www.factorviii-db.org/) via The European Association for Haemophilia and Allied Disorders. Any cases with mutations corelating to those previously found to be associated with ID in the INSIGHT study (19 mutations) were excluded (9).

Twenty amino acids make up structural proteins. They can be classified into two major groups based on their side chains: non-polar hydrophobic and polar. The polar group is then further classified into 3 subgroups: basic, acidic and polar uncharged (Table I). All information regarding each mutation, including whether the mutation caused an amino acid group change, was recorded; this included the localization (at which domain) of the changed amino acid in the F8 protein.

In silico protein modelling programs were used to interpret the effects of a mutation at the protein level. Almost all such programs give probability scoring ranging from benign to pathogenic for the effect of a certain mutation. Each have a unique algorithm and cut-off value. In this study, we used Combined Annotation Dependent Depletion (CADD), Rare Exome Variant Ensemble Learner (REVEL), Mendelian Clinically Applicable Pathogenicity (M-CAP) and Deleterious Annotation using Neural Networks (DANN) scores to find a significant cut-off value and specific markers indicative of ID (10-13).

\section{Statistical Analysis}

Statistical package IBM SPSS version 25 (IBM Corp., Armonk, NY, USA) was used to analyze the data. The chi-squared test was used to compare differences in the categorical data between groups and $p<0.05$ was regarded as statistically significant. The two-sample independent t-test was used for the numeric data; and then to define a cut-off value ROC analysis was performed in data found statistically significant. All hypothesis tests were carried out based on a 0.05 significance level.

\section{Results}

All recorded cases prior to May 2019 in the F8 gene variation database (http://www.factorviii-db.org/) were screened and 3,248 different cases with 954 different missense mutations possessing the necessary study criteria were recruited. The missense mutations recruited were in 607 different points in the cDNA of the F8 gene. Of the total 3,248 cases, 3,078 contained information regarding the clinical severity of the disease, and 2,251 had information regarding ID. When cases were evaluated based on clinical severity, 1.717 (55.8\%) had mild HA, 639 (20.8\%) moderate, and $722(23.5 \%)$ severe HA. Thus, of all 3,078 cases, $76.5 \%$ $(n=2,356)$ were considered mild or moderate HA. There were 2,251 cases possessing information regarding inhibitor status. From among these, 157 (7\%) cases were recorded as being positive for ID against the FVIII protein. There were 2,207 cases in which information about both clinical phenotype and inhibitor information was present. Of these, $153(6.9 \%)$ had been reported as being inhibitor positive. Using a 4-group classification (including subgroups of amino acids) (Table I), evaluation according to whether substitution caused changes in the amino acid class was 
Table I. Amino acid classification according to their side chains

\begin{tabular}{|l|l|}
\hline Amino acid class & Amino acids \\
\hline Class 1: Non-polar Hydrophobic & Ala, Val, Leu, Ile, Met, Pro, Phe \\
\hline Class 2a: Polar uncharged & Ser, Tyr, Asn, Gln, Cys, Thr, His, Gly \\
\hline Class 2b: Acidic & Asp, Glu \\
\hline Class 2c: Basic & Lys, Arg \\
\hline
\end{tabular}

made. Results showed that mutations causing no amino acid group changes had a higher association with ID $(p=0.012)$. The ID rate was $8.9 \%(n=67 / 755)$ in cases with mutations resulting in no changes in amino acid groups, while it was $6.0 \%(n=90 / 1,496)$ in cases with mutations causing amino acid group changes. The difference between these two groups increased when the cases differed in terms of clinical severity. In mild HA, 9.8\% ( $n=44 / 451)$ of patients with mutations causing no amino acid group changes had inhibitors while inhibitors were seen in only $4.8 \%(n=34 / 709)$ of patients with mutations causing amino acid group changes $(p=0.001)$. In moderate and severe HA patients, no significant difference between these two groups was observed (Table II).

Using 2 group classifications for the evaluation of missense mutations according to whether substitution caused changes in the amino acid class, it was observed that mutations causing no amino acid changes had a higher relationship with ID in mild HA cases than those mutations resulting in amino acid changes $(p=0.001)$. Among mild HA patients, $7.9 \%$ ( $n=70 / 883$ ) with mutations causing no amino acid group changes showed ID. This rate, however, was only $2.9 \%$ in those patients with mutations leading to amino acid group changes. In moderate and severe HA, no statistical difference was detected between the groups, similar to the 4-group classification (Table II).

In this study, associations between affected domains and ID were also investigated. There was no ID in patients having mutations affecting $A 1, A 2, A 3$ and SP domains of the protein. Those patients with mutations affecting C1 (11.4\%) and C2 (10.8\%) domains had the highest risk. The rates were $7.3 \%, 7.2 \%$, and $3.1 \%$ in patients with mutations affecting $A 2, A 3$ and $A 1$ domains respectively.

After combining the information in this study; including clinical severity and amino acid group changes, associations between affected domains and IDs were reevaluated. Using the 4-group classification, in mild HA patients with mutations causing no amino acid group changes affecting $A 2, A 3$ and $C 2$ domains, ID risk was found to be higher than for those patients with mutations leading to amino acid group changes; ID rates were $10.4 \%(n=18 / 173)$ versus $3.7 \%(n=7 / 188)$ for the A2 domain $(p=0.012), 7.6 \%(n=7 / 92)$ versus $1.4 \%(n=2 / 142)$ for the $A 3$ domain $(p=0.016)$ and $20.8 \%(n=11 / 53)$ versus $7.3 \%(n=7 / 96)$ for the $C 2$ domain $(p=0.016)$ (Table III).

To identify a significant cut-off value and a specific marker indicative of ID, we used CADD, REVEL, M-CAP, and DANN scores; breaking down the cases according to clinical phenotype. No significant association between M-CAP or DANN scores and ID was noted. However, in mild/ moderate HA cases, CADD and REVEL scores were found to be associated with ID. To establish a cut-off value indicative of ID in mild/moderate HA cases, ROC analysis was applied. For CADD and REVEL scores, the area under the curve (Table IV) (Figure 1) was found to be significant.

\section{Discussion}

Herein, we showed for the first time in the literature that, in mild $H A$, there could be significant relationship between whether a mutation causes amino acid group change and its impact on ID.

It is commonly regarded that the degree of severity of the disease represents an important factor for the occurrence of ID; and in severe hemophilia, factors affecting ID are wellknown. These include familial predisposition, mutation type, human leucocyte antigen class II polymorphism, immunological factors, and environmental factors such as surgery and trauma (14-16). However, it is considered that genetics play the most crucial role when it comes to mutation type. The treatment protocols for patients are designed only after considering all these factors.

It has been reported that there is approximately a 3-10\% risk of ID in mild hemophilia cases. Most of the mild HA cases with inhibitors have found to be missense mutations. However, in these cases, no predictive data related to features of the mutations in the $F 8$ gene in terms of ID has been reported (17). In this study, based on information obtained from the F8 variant database, the risk of ID in mild HA cases was determined to be approximately $7 \%$. 
Table II. Relationship between inhibitor development and changes in amino acid class due to missense mutation. In this table, statistics in the four-group classification indicate changes between non-polar hydrophobic, basic, acidic and polar uncharged amino acids and statistics in the two-group classification indicate changes between non-polar hydrophobic and polar amino acids.

\begin{tabular}{|c|c|c|c|c|}
\hline \multirow{2}{*}{\multicolumn{2}{|c|}{ All patients $(n=2,251)$}} & \multicolumn{2}{|c|}{ Changes in amino acid class (Four-group classification) } & \multirow{2}{*}{ p-value } \\
\hline & & No (\%) & Yes (\%) & \\
\hline \multirow{2}{*}{ 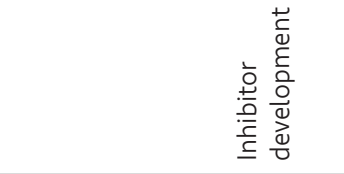 } & No & $688(91.1)$ & $1,406(94)$ & \multirow[b]{2}{*}{0.012} \\
\hline & Yes & $67(8.9)$ & $90(6.0)$ & \\
\hline \multirow{2}{*}{\multicolumn{2}{|c|}{ In mild cases $(n=1,160)$}} & \multicolumn{2}{|c|}{ Changes in amino acid class (Four-group classification) } & \multirow{2}{*}{ p-value } \\
\hline & & No (\%) & Yes (\%) & \\
\hline \multirow{2}{*}{ 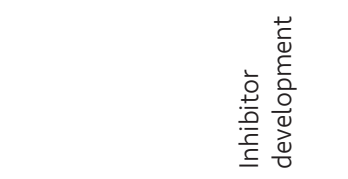 } & No & $407(90.2)$ & $675(95.2)$ & \multirow[b]{2}{*}{0.001} \\
\hline & Yes & $44(9.8)$ & $34(4.8)$ & \\
\hline \multirow{2}{*}{\multicolumn{2}{|c|}{ In mild cases $(n=1,160)$}} & \multicolumn{2}{|c|}{ Changes in amino acid class (Two-group classification) } & \multirow{2}{*}{ p-value } \\
\hline & & No $(\%)$ & Yes (\%) & \\
\hline \multirow{2}{*}{ 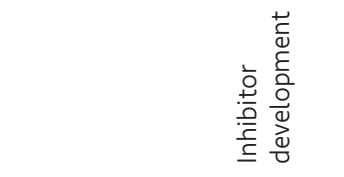 } & No & $813(92.1)$ & $269(97.1)$ & \multirow[b]{2}{*}{0.001} \\
\hline & Yes & $70(7.9)$ & $8(2.9)$ & \\
\hline \multirow{2}{*}{\multicolumn{2}{|c|}{ In moderate cases ( $n=496)$}} & \multicolumn{2}{|c|}{ Changes in amino acid class (Four-group classification) } & \multirow{2}{*}{ p-value } \\
\hline & & No (\%) & Yes (\%) & \\
\hline \multirow{2}{*}{ 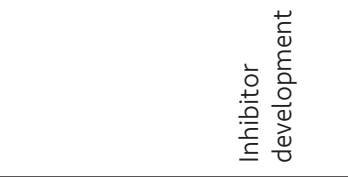 } & No & $123(96.1)$ & $345(93.8)$ & \multirow[b]{2}{*}{0.322} \\
\hline & Yes & $5(3.9)$ & $23(6.3)$ & \\
\hline \multirow{2}{*}{\multicolumn{2}{|c|}{ In moderate cases ( $n=496)$}} & \multicolumn{2}{|c|}{ Changes in amino acid class (Two-group classification) } & \multirow{2}{*}{ p-value } \\
\hline & & No (\%) & Yes (\%) & \\
\hline \multirow{2}{*}{ 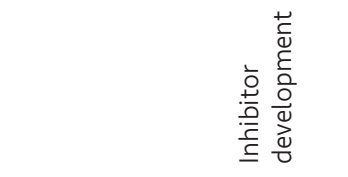 } & No & $326(94.8)$ & $142(93.4)$ & \multirow[b]{2}{*}{0.549} \\
\hline & Yes & $18(5.2)$ & $10(6.6)$ & \\
\hline \multirow{2}{*}{\multicolumn{2}{|c|}{ In severe cases $(n=551)$}} & \multicolumn{2}{|c|}{ Changes in amino acid class (Four-group classification) } & \multirow{2}{*}{ p-value } \\
\hline & & No (\%) & Yes (\%) & \\
\hline \multirow{2}{*}{ 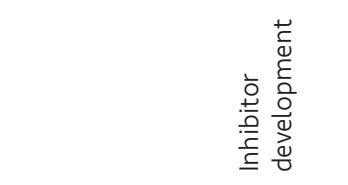 } & No & $135(89.4)$ & $369(92.3)$ & \multirow[b]{2}{*}{0.286} \\
\hline & Yes & $16(10.6)$ & $31(7.8)$ & \\
\hline \multirow{2}{*}{\multicolumn{2}{|c|}{ In severe cases $(n=551)$}} & \multicolumn{2}{|c|}{ Changes in amino acid class (Two-group classification) } & \multirow{2}{*}{ p-value } \\
\hline & & No $(\%)$ & Yes (\%) & \\
\hline$\stackrel{+}{\check{\Sigma}}$ & No & $352(91.9)$ & $152(90.5)$ & \\
\hline 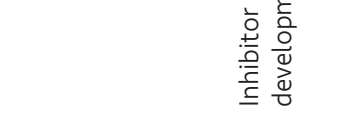 & Yes & $31(8.1)$ & $16(9.5)$ & 0.322 \\
\hline
\end{tabular}




\begin{tabular}{|c|c|c|c|c|}
\hline \multirow{2}{*}{\multicolumn{2}{|c|}{$\begin{array}{l}\text { Domain A2 } \\
\text { In mild cases }(n=361)\end{array}$}} & \multicolumn{3}{|c|}{ Changes in amino acid class (Four-group classification) } \\
\hline & & No $(\%)$ & Yes (\%) & p-value \\
\hline \multirow{2}{*}{ 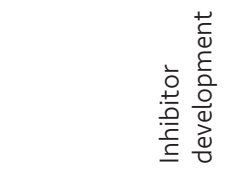 } & No & $155(89.6)$ & $181(96.3)$ & \multirow{2}{*}{0.012} \\
\hline & Yes & $18(10.4)$ & $7(3.7)$ & \\
\hline \multicolumn{2}{|c|}{ In moderate cases $(n=98)$} & No & Yes & p-value \\
\hline \multirow{2}{*}{ 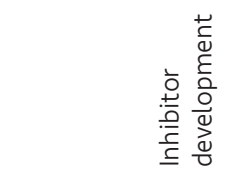 } & No & $31(93.9)$ & $62(95.4)$ & \multirow[b]{2}{*}{0.759} \\
\hline & Yes & $2(6.1)$ & $3(4.6)$ & \\
\hline \multicolumn{2}{|c|}{ In severe cases $(n=112)$} & No & Yes & p-value \\
\hline \multirow{2}{*}{ 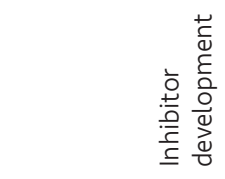 } & No & $38(88.4)$ & $62(89.9)$ & \multirow{2}{*}{0.805} \\
\hline & Yes & $5(11.6)$ & $7(10.1)$ & \\
\hline \multicolumn{2}{|l|}{ Domain A3 } & \multicolumn{3}{|c|}{ Changes in aminoacid class (Four groups classification) } \\
\hline \multicolumn{2}{|c|}{ In mild cases $(n=234)$} & No & Yes & p-value \\
\hline \multirow{2}{*}{ 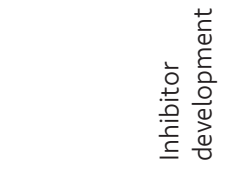 } & No & $85(92.4)$ & $140(98.6)$ & \multirow{2}{*}{0.016} \\
\hline & Yes & $7(7.6)$ & $2(1.4)$ & \\
\hline \multicolumn{2}{|c|}{ In moderate cases $(n=110)$} & No & Yes & p-value \\
\hline \multirow{2}{*}{ 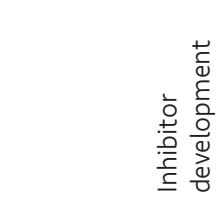 } & No & $32(97.0)$ & $74(96.1)$ & \multirow{2}{*}{0.824} \\
\hline & Yes & $1(3)$ & $3(3.9)$ & \\
\hline \multicolumn{2}{|c|}{ In severe cases $(n=101)$} & No & Yes & p-value \\
\hline \multirow{2}{*}{ 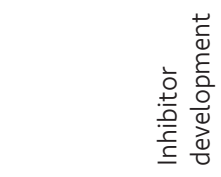 } & No & $16(84.2)$ & $67(81.7)$ & \multirow[b]{2}{*}{0.797} \\
\hline & Yes & $3(15.8)$ & $15(18.3)$ & \\
\hline \multicolumn{2}{|l|}{ Domain C2 } & \multicolumn{3}{|c|}{ Changes in aminoacid class (Four groups classification) } \\
\hline \multicolumn{2}{|c|}{ In mild cases $(n=149)$} & No & Yes & $\mathrm{p}$-value \\
\hline
\end{tabular}


Table III. contiuned

\begin{tabular}{|c|c|c|c|c|}
\hline \multirow{2}{*}{ 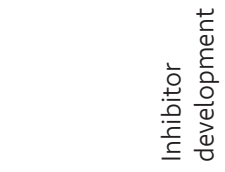 } & No & $42(79.2)$ & $89(92.7)$ & \multirow{2}{*}{0.016} \\
\hline & Yes & $11(20.8)$ & $7(7.3)$ & \\
\hline \multicolumn{2}{|c|}{ In moderate cases ( $n=62$ ) } & No & Yes & $\mathrm{p}$-value \\
\hline \multirow{2}{*}{ 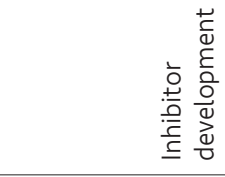 } & No & $5(83.3)$ & 49 (87.5) & \multirow{2}{*}{0.772} \\
\hline & Yes & $1(16.7)$ & $7(12.5)$ & \\
\hline \multicolumn{2}{|c|}{ In severe cases $(n=64)$} & No & Yes & $\mathrm{p}$-value \\
\hline \multirow{2}{*}{ 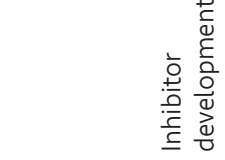 } & No & $8(100)$ & $52(92.9)$ & \multirow{2}{*}{0.435} \\
\hline & Yes & $0(0)$ & $4(6.3)$ & \\
\hline
\end{tabular}

Table IV. (A) The comparison between interpretation scores and inhibitor development and (B) cut-off values of interpretation scores and their sensitivity/specificity in those patients with mild/moderate HA.

\begin{tabular}{|c|c|c|c|c|c|c|c|}
\hline $\begin{array}{l}\text { (A) } \\
\text { Scores }\end{array}$ & Inhibitor development & $\mathbf{n}$ & Mean & \multicolumn{2}{|c|}{$\begin{array}{l}\text { Standard } \\
\text { deviation }\end{array}$} & \multicolumn{2}{|l|}{ p-value } \\
\hline \multirow{2}{*}{ CADD phred } & No & 1,550 & 27.87 & \multicolumn{2}{|l|}{ \pm 5.23} & \multirow{2}{*}{\multicolumn{2}{|c|}{0.000}} \\
\hline & Yes & 106 & 29.80 & \pm 4.05 & & & \\
\hline \multirow{2}{*}{ CADD raw } & No & 1,550 & 5.74 & \pm 1.51 & & \multirow{2}{*}{\multicolumn{2}{|c|}{0.000}} \\
\hline & Yes & 106 & 6.41 & \pm 1.15 & & & \\
\hline \multirow{2}{*}{ REVEL score } & No & 1,550 & 0.84 & \pm 0.11 & & \multirow{2}{*}{\multicolumn{2}{|c|}{0.000}} \\
\hline & Yes & 106 & 0.89 & \multicolumn{2}{|l|}{ \pm 0.08} & & \\
\hline $\begin{array}{l}\text { (B) } \\
\text { Scores }\end{array}$ & $\begin{array}{l}\text { Susceptible to inhibitor } \\
\text { development if greater than or } \\
\text { equal to }\end{array}$ & Sensitivity & Specificity & LR+ & LR- & $\begin{array}{l}\text { Area under } \\
\text { the curve }\end{array}$ & p-value \\
\hline CADD phred & 28.05 & 0.65 & 0.60 & 1,599 & 0.585 & 0.634 & 0.000 \\
\hline CADD raw & 6.11 & 0.65 & 0.61 & 1,647 & 0.573 & 0.640 & 0.000 \\
\hline REVEL score & 0.87 & 0.65 & 0.62 & 1,681 & 0.566 & 0.642 & 0.000 \\
\hline
\end{tabular}

The risk factors for ID in severe hemophilia are wellknown; however in mild hemophilia, these influencing factors remain unclear. Although a family history of ID and treatment-related factors have been reported as risk factors, the specific mutation features have not been closely investigated (18). Certain F8 gene missense mutations contribute to the development of inhibitors in patients with mild hemophilia, sometimes up to levels observed in patients with the severe form of HA disease $(7,14)$. This association with F8 mutations was first demonstrated in a cohort study by Eckhardt et al. (16). In that study of 128 patients with mild $\mathrm{HA}$ and 10 patients with moderate $\mathrm{HA}$, of the ten subjects that developed inhibitors, eight carried the Arg593Cys mutation. Eckhardt et al. (9), further investigated the results of the INSIGHT study, cross referencing a registry involving 34 hemophilia treatment centers across 11 countries. In their study, from among a total of 214 different F8 missense mutations, 19 were found to be associated with 

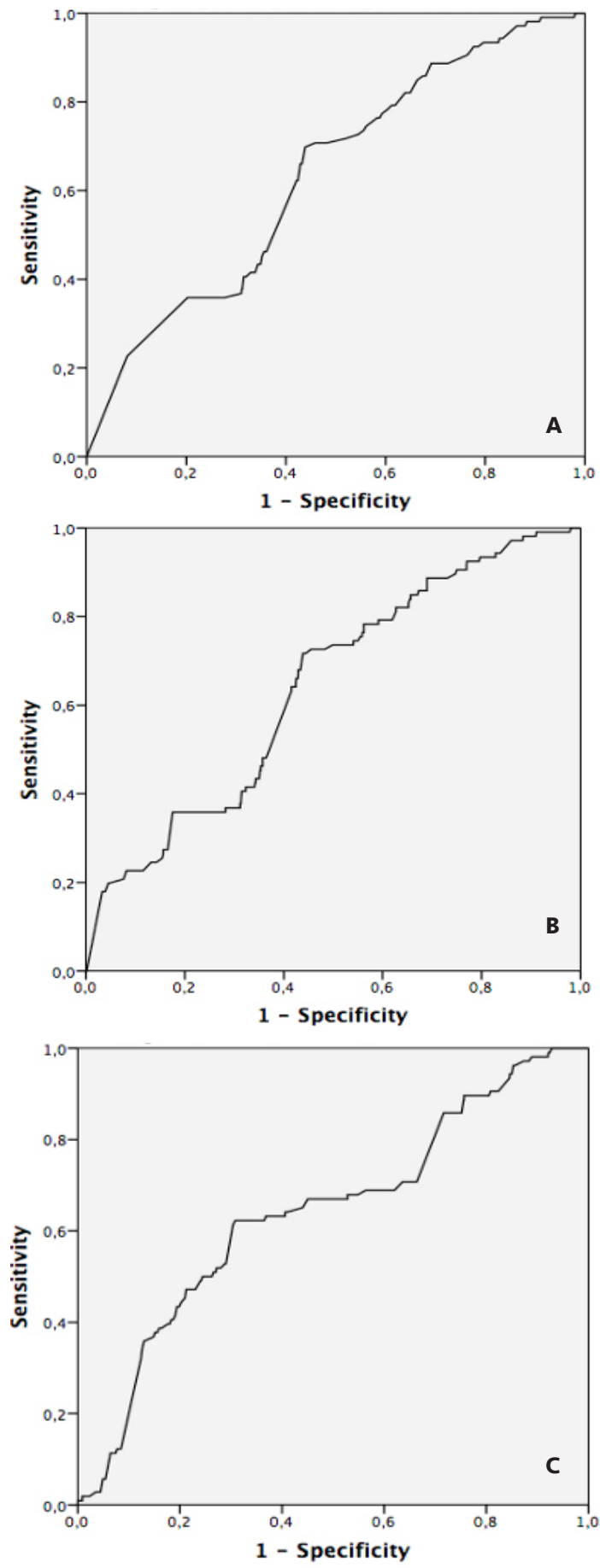

Figure 1. ROC analysis of A) CADD phred score B) CADD raw score C) REVEL score.

CADD: Combined Annotation Dependent Depletion, REVEL: Rare Exome Variant Ensemble Learner
ID (The INSIGHT Study). However, to date, no association between the different features of missense mutations and ID risk had been studied.

In this study, we detected 2 features offering potential for the prediction of inhibitor risk development in mild HA cases caused by missense mutation. Firstly; if the missense mutation found in a mild HA patient does not cause amino acid group change, ID risk increases. Secondly; missense mutations causing no amino acid group change which also affect the $A 2, A 3$ and $C 2$ domains of the $F 8$ protein lead to a statistically significant higher risk of ID.

A number of previous studies have confirmed the relationships between 19 missense mutations reported in the INSIGHT study and ID. In the study by Kempton et al. (19), 3 different missense mutations (R593C, R2150H, and N1922S) were found to be causative for ID in a cohort that included 18 mild/moderate HA patients. Two (R593C and $\mathrm{R} 2150 \mathrm{H}$ ) of these three mutations were also reported in the INSICHT study, while one mutation (N1922S) was not. We consider that as the N1922S mutation does not cause amino acid group change, it could, therefore, be a factor for ID (19). lioka et al. (20) reported a mild HA patient developing high titers of inhibitors. This patient had a missense mutation, c.3780C > G (p.D1241E), and required a long-term treatment protocol. They argued that the high titers of ID was directly related to the long treatment course. Regarding our study results, we consider that in lioka et al.'s (20) patient, ID possibly resulted from a mutation (c.3780C $>$ G) that did not cause amino acid group change.

In this study, we also investigated predictive cut-off values of several mutation pathogenicity scoring systems for ID risk. It is considered that that CADD and REVEL scores can be used for this purpose (Table IV).

\section{Conclusion}

In mild HA patients, the variations of the amino acid group changes in missense mutations, as well as CADD and REVEL scores could offer some utility for the prediction of ID risk. Additionally, missense mutations causing no amino acid group change, and also involving $A 2, A 3$ and $C 2$ domains of the FVIII protein are considered to lead to the highest risk of ID. Due to the retrospective design of our study, information regarding the treatment regimen of patients presented was severely limited. It is considered that further prospective studies, including treatment regimens, are required to fully evaluate the utility of the ID prediction hypotheses put forward in this study. 


\section{Ethics}

Ethics Committee Approval: The article was made using data pulled from a database (openaccess).

Informed Consent: The article was made using data pulled from a database (openaccess).

Peer-review: Externally peer-reviewed.

\section{Authorship Contributions}

Concept: E.I., Design: K.K., F.Ö., T.A., Data Collection or Processing: H.M., B.A., Analysis or Interpretation: T.K., Reviewing of Manuscript: F.Ö., T.A.

Conflict of Interest: No conflict of interest was declared by the authors.

Financial Disclosure: The authors declared that this study received no financial support.

\section{References}

1. Saint-Remy J-MR, Lacroix-Desmazes S, Oldenburg J. Inhibitors in haemophilia: pathophysiology. Haemophilia 2004; 10(Suppl 4):146-51.

2. Schwaab R, Brackmann $H H$, Meyer $C$, et al. Haemophilia A: mutation type determines risk of inhibitor formation. Thromb Haemost 1995; 74:1402-6.

3. Pavlova A, Delev D, Lacroix-Desmazes S, et al. Impact of polymorphisms of the major histocompatibility complex class II, interleukin-10, tumor necrosis factor-alpha and cytotoxic T-lymphocyte antigen-4 genes on inhibitor development in severe hemophilia A.Thromb Haemost 2009; 7:2006-15.

4. Peyvandi F, Mannucci PM, Garagiola I, et al. A randomized trial of factor vIII and neutralizing antibodies in hemophilia A. N Eng I Med 2016; 374:2054-64.

5. Gouw SC, van den Berg HM, Fischer K, et al. Intensity of factor VIII treatment and inhibitor development in children with severe hemophilia A: the RODIN study. Blood 2013; 121:4046-55.

6. Gouw SC, van der Bom JG, Marijke van den Berg H. Treatmentrelated risk factors of inhibitor development in previously untreated patients with hemophilia A: the CANAL cohort study. Blood 2007; 109:4648-54

7. Oldenburg J, El-Maarri O, Schwaab R. Inhibitor development in correlation to factor VIII genotypes. Haemophilia 2002; 8(Suppl 2):23-9.
8. Oldenburg I, Pavlova A. Genetic risk factors for inhibitors to factors VIII and IX. Haemophilia 2006; 12(Suppl 6):15-22.

9. Eckhardt $\mathrm{CL}$, van Velzen $\mathrm{AS}$, Peters $\mathrm{M}$, et al. Factor VIII gene (F8) mutation and risk of inhibitor development in nonsevere hemophilia A. Blood 2013; 122:1954-62.

10. Kircher M, Witten DM, Jain P, O'Roak BJ, Cooper GM, Shendure J. A general framework for estimating the relative pathogenicity of human genetic variants. Nat Genet 2014; 46:310-5.

11. Quang D, Chen Y, Xie X. DANN: a deep learning approach for annotating the pathogenicity of genetic variants. Bioinformatics (Oxford, England) 2015; 31:761-3.

12. Ioannidis NM, Rothstein JH, Pejaver V, et al. REVEL: an ensemble method for predicting the pathogenicity of rare missense variants. Am J Human Genet 2016; 99:877-85.

13. Jagadeesh KA, Wenger AM, Berger MJ, et al. M-CAP eliminates a majority of variants of uncertain significance in clinical exomes at high sensitivity. Nat Genet 2016; 48:1581-6.

14. d'Oiron R, Pipe SW, Jacquemin M. Mild/moderate haemophilia A: new insights into molecular mechanisms and inhibitor development. Haemophilia 2008; 14(Suppl 3):138-46.

15. Sharathkumar A, Lillicrap D, Blanchette VS, et al. Intensive exposure to factor VIII is a risk factor for inhibitor development in mild hemophilia A. Thromb Haemost 2003; 1:1228-36.

16. Eckhardt $\mathrm{CL}$, Menke $\mathrm{LA}$, van $\mathrm{Ommen} \mathrm{CH}$, et al. Intensive perioperative use of factor VIII and the Arg593-->Cys mutation are risk factors for inhibitor development in mild/moderate hemophilia A. Thromb Haemost 2009; 7:930-7.

17. Sun $\mathrm{HL}$, Chan $\mathrm{S}$, Yenson $\mathrm{P}$, Jackson $\mathrm{S}$. Inhibitor risk stratification and Individualized treatment in patients with nonsevere hemophilia A: a single-ınstitution practice audit. Clin Appl Thromb Hemost 2018; 24:303-9.

18. Hay CR. Factor VIII inhibitors in mild and moderate-severity haemophilia A. Haemophilia 1998; 4:558-63.

19. Kempton $\mathrm{CL}$, Allen $\mathrm{G}$, Hord I, et al. Eradication of factor VIII inhibitors in patients with mild and moderate hemophilia A. Am J Hematol 2012; 87:933-6.

20. lioka F, Shimomura D, Nakamura F, et al. Long-term treatment course of a patient with mild haemophilia A who developed a high titre factor VIII inhibitor. Haemophilia 2014; 20:e402-4. doi: 10.1111/hae.12491. 